Sumner, E., Pratt, M.L. \& Hill, E.L. (in press). Examining the cognitive profile of children with Developmental Coordination Disorder. Research in Developmental

Disabilities. Elsevier

\title{
Examining the cognitive profile of children with Developmental Coordination Disorder
}

\author{
Emma Sumner $^{\mathrm{a}^{*}}$, Michelle L. Pratt ${ }^{\mathrm{b}}$, Elisabeth L. Hill ${ }^{\mathrm{a}}$
}

\begin{abstract}
Affiliations: ${ }^{a}$ Department of Psychology, Goldsmiths, University of London, UK; and ${ }^{\mathrm{b}}$ Psychological Medicine Unit, Chelsea and Westminister Hospital, UK
\end{abstract}

\section{*Corresponding author:}

Elisabeth Hill

Department of Psychology

Goldsmiths, University of London

New Cross

London

United Kingdom

SE14 6NW

Email: e.hill@gold.ac.uk

Short title: Cognitive profile, Developmental Coordination Disorder 


\section{Examining the cognitive profile of children with Developmental Coordination Disorder}

Background. While primarily a motor disorder, research considering the cognitive abilities in children with Developmental Coordination Disorder (DCD) is limited; even though these children often struggle academically.

Aims. The present study aimed to characterise the IQ profile of children with and without DCD, and to identify whether children with DCD exhibit specific cognitive weaknesses.

Methods and Procedures. 104 children participated in the study. Fifty-two children (mean age, 9 years) with a diagnosis of DCD were matched to 52 typically-developing children by age and gender. Cognitive ability was assessed using the Wechsler Intelligence Scale for Children (WISC-IV).

Outcomes and Results. Children with DCD performed poorer than their peers on processing speed and working memory measures. Individual analyses revealed varied performance in the DCD group across all cognitive indices, despite displaying Full-Scale IQs in the typical range. Discriminant function analyses show processing speed and working memory performance predicted only $23 \%$ of between-group variability.

Conclusions. Children with DCD present with a heterogeneous cognitive profile, lending support to individual case analyses in research and when designing educational assistance plans. The motorically-demanding nature of the WISC-IV processing speed tasks raises specific concerns about using this index of the IQ assessment in this population. Research and practical implications are raised.

\section{What this paper adds?}

Intellectual (cognitive) abilities are assessed in research and clinical practice. A link between cognitive and motor skill is often reported in the typically-developing literature, but little is known about the cognitive profile of children with Developmental Coordination Disorder (DCD). This paper provides a comprehensive assessment of a range of cognitive abilities, using the popular Wechsler scales. For the first time, it shows that children with DCD perform poorly on only the processing speed and working memory measures, in comparison to their peers. However, the motor demands of processing speed tasks can in part explain these results. Moreover, a heterogeneous cognitive profile was identified in the DCD group. Analysis of individual differences shows that children with DCD do not present with a distinct IQ profile. This suggests that attention should be paid to individual case analyses in order to understand the relationship between motor skill and IQ. The paper addresses the risk of identifying DCD on the basis of an IQ profile, and encourages all practitioners to adhere to the standard diagnostic procedures identified in the DSM-5. 
Keywords. Cognitive ability, Developmental Coordination Disorder (DCD), diagnosis, intelligence, motor impairment

\section{Highlights}

- The WISC-IV provides a detailed account of the cognitive profile of children with DCD.

- The DCD group, overall, present with weaknesses in processing speed and working memory.

- Within-group analyses depict a heterogeneous cognitive profile in the DCD group.

- Motor demands in the WISC-IV PSI are a potential confound, but performance in this area may highlight the need for further exploration of motor difficulties.

- The DCD group cannot be differentiated from their peers solely on a cognitive profile. 


\section{Introduction}

Intellectual functioning is assessed across clinical, educational and research settings. It is often examined during the diagnostic process of neurodevelopmental disorders to eliminate global developmental delay; and to confirm that any specific behavioural characteristics of a disorder are not better explained by low measured IQ (Castles, Kohnen, Nickels \& Brock, 2014). For example, the diagnostic criteria for Developmental Coordination Disorder (DCD) specify that movement difficulties must be below the level expected for the individual's age and cannot be accounted for by intellectual disability (APA, 2013). Practitioners typically administer a battery of tests examining intellectual abilities to guide their overall assessment of a child with a movement difficulty. Moreover, in education practice, identifying specific cognitive profiles (i.e. strengths and weaknesses) can help to guide strategies for educational assistance and provide a way of monitoring intervention outcomes.

Smits-Engelsman and Hill (2012) recognised that a cut-off IQ score of 70 to 80 is often used when diagnosing DCD in clinical practice, or confirming a diagnosis of DCD for research purposes: consistent with the DSM-5 diagnostic criteria. Yet, surprisingly, a recent review of 176 studies published on DCD in the last 5 years, identified that only $30 \%$ specifically tested IQ (Smits-Engelsman, Schoemaker, Delabastita, Hoskens \& Geuze, 2015). A large proportion of studies failed to control for IQ and instead presume that recruitment from a mainstream school means cognitive abilities will be within the average range. Thus, research appears to lend little focus to the role of IQ in DCD. However, the literature suggests that cognitive development and motor performance are intertwined and follow a similar developmental trajectory (Anderson, Anderson, Northam, Jacobs \& Catroppa, 2001; Smits-Engelsman \& Hill, 2012). Early theories by Piaget (1952) also stressed that the cognitive and motor systems work together from a young age, as children learn about their surroundings from interacting with, and manipulating objects; and more recently, a systematic review of research on typically-developing children aged 4-16 years, highlighted that better motor skill was commonly associated to stronger performance in cognitive tasks (van der Fels et al., 2015). Therefore, an understanding of this relationship in DCD may prove to be important for education practice and the diagnostic process. Especially since the difficulties experienced by children with DCD often extend beyond motor ability, and problems in academic achievement are often reported (Dewey, Kaplan, Crawford \& Wilson, 2002; Kirby \& Sugden, 2007). Yet, despite being a condition with an estimated prevalence between 2-5\% (Kirby \& Sugden, 2007; Lingham, Hunt, Golding, Jongmans, \& Emond, 2009), to our knowledge, no study has systematically investigated the full range of cognitive abilities and whether specific IQ/cognitive profiles exist in a DCD population.

Intelligence scales, such as the widely used Wechsler scales (Wechsler, 2003), provide a comprehensive assessment of cognitive abilities. The Wechsler Intelligence Scale for Children, Fourth Edition (WISC-IV, 2003) provides a measure of Full Scale IQ (FSIQ), which is derived from performance on ten subtests that comprise four key indices: Verbal Comprehension (VCI), Perceptual Reasoning (PRI), Working Memory (WMI) and Processing Speed (PSI). Using these indices, or at the subtest level, researchers have attempted to delineate the pattern of cognitive function and its relation to the behavioural characteristics often shown in many neurodevelopmental disorders often seen in the 
classroom, such as: Autism Spectrum Disorder (ASD, Charman et al., 2010; Mayes \& Calhoun, 2008; Oliveras-Rentas, Kenworthy, Roberson, Martin, \& Wallace, 2012), dyslexia (Moura, Simoes, \& Pereira, 2014), or Attention Deficit-Hyperactivity Disorder (ADHD; Hagberg, Miniscalco \& Gillberg, 2010; Parke, Thaler, Etcoff \& Allen, 2015). In support of the WISC-IV, practitioners argue that its most beneficial feature is to generate cognitive profiles to help determine educational placement (Pfeiffer, Reddy, Kletzel, Schmelzer \& Boyer, 2012).

Studies that have partly assessed IQ/cognitive ability in DCD, using the Wechsler scales, present a mixed and inconclusive account. In comparison to typically-developing children and those with known attention problems, children with DCD have been identified as performing lower on FSIQ (Kaplan, Wilson, Dewey \& Crawford, 1998). However, the authors did not consider performance at the index or subtest level. While general processing deficits are often reported in a DCD population (Piek, Dyck, Francis \& Conwell, 2007; Wilson \& McKenzie, 1998), Loh, Piek and Barrett (2011) found no difference between children with DCD, children with ADHD, and a typically-developing control group in processing speed (using WISC-IV, PSI). This finding should, however, be considered as exploratory due to the small sample size (eleven participants per group). In addition, Loh et al. (2011) reported lower PRI in children with DCD in comparison to VCI, but, of note, the authors opted to use the short form of the WISC-IV, thus excluding WMI and one subtest from each of the VCI and PRI.

In a group known to have a heterogeneous behavioural profile, a more thorough assessment of individual differences is required, considering whether a profile of strengths and weaknesses is present across the cognitive indices. Therefore the purpose of the present study was to clarify two questions at a group and individual level, using a popular method of assessment for research and clinical purposes (WISC-IV; Wechsler, 2003):

(1) Do children with DCD present with notable weaknesses in any of the four WISC-IV indices (or subtests) in comparison to age-matched controls?

(2) Does one (or more) of these area(s) of weakness appear primary to DCD?

\section{Method}

\subsection{Participants}

Fifty-two children with DCD (36 boys) were included in the study, ranging from 7-14 years. Children with DCD were compared to 52 typically-developing (TD) children (36 boys) matched by age and gender. All children had a FSIQ $>70$ on the WISC-IV (Wechsler, 2003).

\subsubsection{DCD group}

Children with DCD were recruited through primary schools in $\mathrm{XXX}^{1}$, support groups and by advertising through a charitable foundation, the $\mathrm{XXX}^{1}$. Children already had a primary (and single) diagnosis of DCD by a clinician and were assessed for the study to confirm that they met the formal diagnostic criteria set out in the DSM-5 (APA, 2013). All children performed below the $10^{\text {th }}$ percentile ( 45 children below the $5^{\text {th }} ; 86 \%$ ) on the Movement Assessment Battery for Children - Second Edition (MABC-2; Henderson, Sugden

\footnotetext{
${ }^{1}$ Removed for blinding purposes
} 
\& Barnett, 2007). Parents identified that their child's motor difficulties impacted significantly on activities of daily living on a background screening questionnaire; often reporting that their child had difficulty dressing and using cutlery, and a high number reported handwriting difficulties. Parents also provided information regarding any additional diagnoses or medical conditions. Children were excluded if they were identified as having any additional diagnoses, such as ADHD, ASD or dyslexia, a neurological condition or other medical condition which might explain the child's motor impairment.

\subsubsection{Typically-developing (TD) control group}

The control group was recruited through local authority primary schools in $\mathrm{XXXX}^{1}$. To confirm that the children assigned to this group did not display signs of a motor difficulty, they were tested individually on the MABC-2 test (Henderson et al., 2007). Participants in this group scored at or above the $25^{\text {th }}$ percentile. Parents were asked for the same background information as those in the DCD group. Children with a diagnosis of any sort (psychological, neurological or medical) were excluded from the study. See Table 1 for characteristics of the DCD and TD groups.

Table 1. Mean (SD) for age and motor performance scores for DCD and TD groups.

\begin{tabular}{lcc}
\hline & DCD $(n=52)$ & TD $(n=52)$ \\
\hline Age in years & $9.16(2.19)$ & $9.33(1.50)$ \\
MABC-2 standard score & $3.53(1.69)$ & $10.80(1.91)^{*}$ \\
MABC-2 test percentile & $2.94(3.15)$ & $58.38(20.65)^{*}$ \\
\hline
\end{tabular}

Note. ${ }^{*} p<.05$. MABC-2; Movement Assessment Battery for Children - second edition, standard score $M 10, S D 3$

\subsection{Measures}

Cognitive ability was measured using the WISC-IV (UK norms; Wechsler, 2003). FSIQ is the sum of the four indices: VCI, PRI, WMI, and PSI, all of which are converted to standard scores ( $M$ 100, $S D$ 15). Ten subtests are split across the four indices. Subtest items are age-appropriate and raw scores are converted to scaled scores (M 10, SD 3). Across the specified age groups, reliability (internal consistency) of the indices ranged from .81 to .97 (Wechsler, 2003).

\subsection{Procedure}

Parental consent was obtained for each child. Motor and cognitive assessments were completed either on the same day (with a sufficient break in between) or across two separate days. Children were seen individually in a quiet room either at their school or the research lab.

The dataset was combined from two studies conducted in the same lab, both of which were approved by the $\mathrm{XXX}^{2}$ university ethics committee. Of note, data were collected within a 5 year period and the same versions of the assessments (WISC-IV and MABC-2) were used in both studies. The authors administered both standardized assessments and were fully

\footnotetext{
${ }^{2}$ Removed for blinding purposes
} 
qualified to do so. Both assessments were administered according to the standardized procedures identified in the test manual.

\subsection{Data analysis}

To answer Question 1, repeated measures ANOVAs were used to investigate group differences (between the DCD and TD group) among WISC-IV index and subtest scores. Bonferroni corrections were utilised to control for Type I error. To answer Question 2, the proportion of participants scoring 1SD above or below the mean was calculated (similar to Oliveras-Rentas et al., 2012); then a discriminant function analysis was performed to determine the most parsimonious way to discriminate between groups and classify participants accordingly (based on cognitive performance).

\section{Results}

\subsection{Question 1: Do children with DCD present with notable weaknesses in any of the four WISC-IV indices (or subtests) in comparison to age-matched controls?}

\subsubsection{Between-group comparisons}

Table 2 presents group scores on the WISC-IV measures. Significant group differences were found for FSIQ, $t(102)=-2.22, p=.02$, revealing that children with DCD performed marginally below the TD group. Although a group difference is evident for FSIQ, children with DCD still scored within the average range: close to a mean of 100 .

A repeated measures ANOVA with 'group' as the between-subjects factor revealed no significant differences between the four indices, $F(3,306)=1.44, p=.23, n_{\mathrm{p}}^{2}=.01$, but overall, TD children performed significantly better than children with $\mathrm{DCD}, F(1,102)=6.29$, $p=.01, n_{\mathrm{p}}^{2}=.06$. A significant interaction between the WISC-IV indices and group was found, $F(3,306)=7.58, p<.001, n_{\mathrm{p}}^{2}=.07$. This interaction was analysed using a simple main effects analysis. Statistically, group differences were not found for either the VCI or PRI $(p s>.64)$. Significant effects of group were evident only for the WMI, $F(1,102)=5.99$, $p=.02$, and the PSI, $F(1,102)=22.49, p<.001$. Children with DCD scored below the TD group on both indices.

Table 2. Means (SD) for the WISC-IV Full scale, Index and Subtest scores

\begin{tabular}{lcc}
\hline & DCD $(n=52)$ & TD $(n=52)$ \\
\hline FSIQ & $95.71(12.13)$ & $100.65(10.54)^{*}$ \\
VCI & $98.92(10.95)$ & $97.94(10.18)$ \\
Similarities & $10.98(2.56)$ & $10.32(2.33)$ \\
Vocabulary & $9.84(2.55)$ & $9.61(2.59)$ \\
Comprehension & $8.78(2.30)$ & $9.13(1.76)$ \\
PRI & & \\
Block Design & $99.05(15.16)$ & $99.80(11.19)$ \\
Picture concepts & $9.30(3.05)$ & $10.19(2.60)$ \\
Matrix Reasoning & $10.21(2.97)$ & $10.34(2.15)$ \\
& $9.28(2.92)$ & $9.52(2.28)$ \\
\hline
\end{tabular}




\begin{tabular}{lcc}
\hline WMI & $95.88(12.98)$ & $102.25(13.53)^{*}$ \\
Digit Span & $9.83(2.95)$ & $11.56(3.15)^{*}$ \\
Letter-Number Sequencing & $8.18(2.84)$ & $9.56(2.68)$ \\
& & \\
PSI & $91.31(12.26)$ & $102.21(11.15)^{* *}$ \\
Coding & $7.62(3.22)$ & $10.25(2.28)^{* *}$ \\
Symbol Search & $10.04(7.65)$ & $10.76(2.39)$ \\
& \\
\hline Note. ${ }^{*} p<.05, * * p<.01$ when comparing DCD and TD groups directly. \\
Bonferroni corrections were applied for multiple comparisons on the WISC- \\
IV subtests. FSIQ and Index scores M 100, SD 15; Subtests scores M 10, SD 3 \\
\hline
\end{tabular}

Separate repeated measures ANOVAs were conducted for the subtests belonging to the same WISC-IV index, with 'group' as a between-subjects factor. Due to multiple comparisons across the ten subtests, a Bonferroni correction $(p=.005)$ was applied to these analyses. Significant differences were evident for the three subtests forming the VCI, $F(2$, $204)=27.03, p<.001, n_{p}^{2}=.21$. Contrasts revealed significant differences $(p s<.001)$ between all 3 of these subtests, with similarities being the highest and comprehension scored as the lowest. However, no significant group differences were found, $F(1,102),=.22, p=$ $.64, n_{\mathrm{p}}^{2}=.01$, and there was a non-significant interaction, $F(2,204)=2.37, p=.09, n_{\mathrm{p}}^{2}=.02$. For PRI, non-significant results were found when comparing across the three subtests, $F(2$, $204)=5.45, p=.01, n_{\mathrm{p}}^{2}=.05$; no group differences existed on these tasks, $F(1,102),=.95, p$ $=.33, n_{\mathrm{p}}^{2}=.01$, and a non-significant interaction, $F(2,204)=1.17, p=.31, n_{\mathrm{p}}^{2}=.01$ was reported. These findings suggest that children in the two groups followed a similar pattern of performance on the VCI and PRI tasks.

In contrast, a significant difference was found for performance on the two WMI subtests, $F(1,102)=32.66, p<.001, n_{\mathrm{p}}^{2}=.24$, highlighting that overall children performed better on the Digit Span task than Letter-Number Sequencing. Significant group differences were evident, $F(1,102)=11.54, p=.001, n^{2}{ }_{p}=.10$, showing that TD children scored higher on WMI subtests than children with DCD; although a non-significant interaction was reported, $F(1,102)=.19, p=.66, n_{\mathrm{p}}^{2}=.01$. Univariate analyses were conducted to determine group differences for the two individual subtests. Children with DCD performed significantly worse than TD children on Digit span, $F(1,120)=8.38, p=.004, n_{\mathrm{p}}^{2}=.09$, but not on Letter Number Sequencing, $F(1,102)=7.18, p=.01, n_{\mathrm{p}}^{2}=.07$. Turning to the PSI, a nonsignificant difference was found for performance on the two PSI subtests, $F(1,102)=6.07, p$ $=.02, n_{\mathrm{p}}^{2}=.06$. A significant group difference was evident, $F(1,102),=7.16, p=.002, n_{\mathrm{p}}^{2}=$ .10 , and univariate analyses revealed that this group difference was present only for the Coding subtest, $\mathrm{F}(1,120)=22.98, p<.001, n_{\mathrm{p}}^{2}=.20$; however, a non-significant interaction was reported, $F(1,102)=2.41, p=.12, n_{\mathrm{p}}^{2}=.02$.

As the Coding subtest relies on motor skill (i.e., children have to use a pencil to write down the correct answers as quickly as possible), it was appropriate to determine the extent to which manual dexterity skills impacted on the group difference for this subtest. An analysis of covariance (ANCOVA) revealed that manual dexterity (as measured from the MABC-2) was a significant covariate $(p=.02)$ and that after controlling for this covariate, there were no longer significant group differences on Coding performance, $F(1,101)=1.07$, $p=.30, n_{\mathrm{p}}^{2}=.01$. This suggests that the Coding results were confounded by the manual dexterity difficulties in the DCD group. 


\subsubsection{Within-group comparisons}

Within group analyses revealed significant differences across the WISC-IV indices for DCD, $F(3,153)=6.02, p<.001$, but not TD children, $F(3,153)=2.56, p=.06$. Post hoc analyses revealed significant differences only between VCI and PSI $(p=.004)$, and the PRI and PSI $(p=.008)$, in the DCD group; with poorer performance shown for the PSI.

Within group differences were also found at the subtest level, for DCD, $F(9,432)=$ $5.12, p<.001$, and TD children, $F(9,432)=6.14, p<.001$. Both groups showed a variable profile. Only significant post hoc analyses are reported here $(p s \leq .005)$. The analyses confirmed that for children with DCD, Coding and Letter-Number Sequencing were scored significantly lower than Similarities and Picture Concepts. In contrast, for TD children, Digit Span was significantly higher than Comprehension.

\subsubsection{Individual differences}

Figures 1 and 2 identify the percentage of children that scored more than 1SD above or below the population mean.

\section{[Insert Figure 1 here]}

Notably, there are fairly equivalent proportions scoring above and below the mean in the VCI, PRI and WMI in the DCD group. However, only $2 \%$ of the DCD group scored above 1 SD for the PSI, while $33 \%$ of this group scored below $(\leq 85)$. In addition, it appears that children in the DCD group performed better on the PRI (i.e., more scored above the mean) than the other indices. Notably, the TD group also demonstrated variability across the four indices. A high percentage of TD children scored 1SD above the mean on the LetterNumber Sequencing and Symbol Search. Most apparent was that 56\% of the DCD group scored $\leq 85$ on Coding.

[Insert Figure 2 here]

\subsection{Question 2: Does one (or more) of these area(s) of weakness appear primary to DCD?}

\subsubsection{Predictors of group membership}

As significant group differences were observed only for WMI and PSI, these two indices were included as predictor variables in a discriminant function analysis. Inspection of the discriminate function structure matrix revealed a significant association between groups for the PSI (.97) and WMI (.50), together accounting for $23 \%$ of the variation in the group variable (i.e., whether the participant has a motor impairment or not). The cross validated classification showed that overall $69 \%$ of participants were correctly classified. Subtests that yielded significant group differences were entered into a second analysis, and the structure matrix identified Digit Span (.52) and Coding (.86) as significant predictors. This second model, however, still only explained $30 \%$ of group membership and correctly classified $73 \%$ of participants. 


\section{Discussion}

The present study compared cognitive abilities within and between children with and without DCD. Group findings highlighted that children with DCD scored below TD children on only the WMI and PSI of the WISC-IV (Wechsler, 2003). Closer inspection revealed significantly poorer performance for the DCD group on two specific subtests: Digit Span and Coding. As a group, children with DCD exhibited a more variable pattern of performance across the cognitive domains, in comparison to typically-developing children that collectively presented similar group scores for each index. In particular, children with DCD were noted to perform significantly better on both the VCI and PRI, than PSI. It is, however, important to note that the DCD group performance remained within the average population mean for the indices and subtests.

Studying individual differences provides some support for a weaker working memory and processing speed profile in DCD. It appears that the group difference in PSI is largely driven by the Coding subtest. Over half of this group scored more than one standard deviation (SD) below the population mean on Coding, and nearly 30\% scored below this cut off on Digit Span. Further, although the group means were not statistically different, $40 \%$ of children with DCD scored below the 1SD cut off on Letter-Number Sequencing. It was evident that the DCD group depict a heterogeneous profile in general, with wide variability across all subtests. For example, a quarter of the DCD group scored more than 1SD above the population mean on Similarities, while $23 \%$ of this group scored 1SD below the mean on Comprehension (both in VCI). Similar patterns can be seen in the PRI, where there are roughly equal proportions of children performing above and below the mean on the same subtest. Interestingly, this does not appear specific to DCD, as individual analysis of TD children also demonstrated variable performance on the subtests, despite a solidly average mean on each index when considering the group as a whole. These findings support the need for research to consider individual cases in order to reach well-supported conclusions about the cognitive profile of children with DCD. A heterogeneous profile may be one explanation for the discriminant function analysis revealing that together WMI and PSI, and then Digit Span and Coding, predict only $23 \%$ and $30 \%$ of group membership, respectively. Thus, there is limited evidence of a distinctive IQ profile in children with DCD.

The finding of processing speed difficulties (i.e., Coding) in children with DCD ought to be treated with caution. Coding is confounded by the motorically demanding task of using a pencil to reproduce symbols into empty boxes, accurately and at speed; and, in fact, after controlling for manual dexterity skill, children in the DCD and TD groups were comparable on Coding performance. While researchers report processing deficits on experimental measures (Wilson \& McKenzie, 1997), the nature of the WISC-IV PSI limits conclusions as to whether performance reflects actual cognitive processing, motor difficulties, or both. It is, however, noteworthy that on the Symbol Search task where the motor skill requirements were less than in the Coding task (i.e., putting a line through a yes/no box vs. coping a symbol in a small box), children with DCD performed similarly to TD children. Thus, processing deficits were not consistently identified across tasks.

Research into ASD (Mayes \& Colhoun, 2008) and ADHD (Parke et al., 2015) populations also report poorer performance in PSI and WMI on the WISC, in comparison to the other indices. These two conditions show co-occurrence with DCD (Kirby \& Sugden, 2007). The question then remains as to whether poorer performance in these two domains is a characteristic of developmental disorders more generally, or specific to those with motor difficulties. ASD and ADHD have been linked with executive function difficulties and it is 
possible that the working memory difficulties identified in our DCD sample may be due to this. Certainly a recent review identified that children with DCD present with wide-ranging executive difficulties (Leonard \& Hill, 2015). Similarly, attention problems have been reported in children with DCD (Dewey et al., 2002; Querne et al., 2008) and poor attention may influence working memory performance, as the ability to selectively process/attend to relevant information will inevitably influence how information is retained in working memory. In this sense, generating an IQ profile provides a window into broader cognitive skills, with the potential to flag possible underlying, or closely-related difficulties in other areas of cognitive functioning that clinicians would be wise to follow up.

The PRI findings are particularly interesting. A substantial proportion of children with DCD scored more than one standard deviation above the mean on the PRI and corresponding subtests. First and foremost these findings conflict with the literature of a VCI advantage over PRI (Loh et al., 2011). Moreover, they have important implications for UK educators ascribing to the belief that children with DCD (often referred to as 'dyspraxia') can be diagnosed on the basis of a 'spiky' profile showcasing higher VCI than PRI, and a dip in performance on the Block Design subtest (Grant, 2010). Very few studies specifically report Block Design performance, although notably a few do highlight poor performance on this task in comparison to a control group (Alloway, 2007; Gomez et al., 2015). The present findings, using a large sample, go against this claim of Block Design being a good discriminator of DCD but, as with many studies, replication of the present findings would strengthen the conclusions. Nevertheless, diagnosing DCD solely on the basis of a cognitive profile could be detrimental for three reasons. First, children or adults with DCD may be slipping through the net unnoticed because they fail to meet the IQ profile to which Grant (2010) refers to. Second, individuals may be misdiagnosed with DCD and, as a result, receive inappropriate support. Finally, this method of diagnosis does not complement the standard procedures followed at an international level (Geuze, Schoemaker \& Smits-Engelsman, 2015), or reflect the motor difficulty that is core to the disorder.

Further research and clinical/educational implications can be addressed. The present study provides a platform for discussion about which cognitive measures should be used as part of inclusion criteria in DCD research. Smits-Engelsman et al. (2015) found that few DCD studies control for IQ. Those that use a single subtest could in fact be excluding children with little justification, as a varied profile is shown here in DCD. However it does seem most sensible to use the VCI and PRI tasks to confirm IQ performance in the expected range for research criteria, as WMI and PSI appear most impacted in DCD and therefore a number of children with DCD may be excluded from research on the assumption of low IQ when using predetermined cut-offs, as is often employed (e.g., 1SD below the mean). From a clinical and educational assessment perspective, given the impact of PSI on FSIQ, using the WISC-IV General Ability Index might be a more robust measure of intellectual functioning than FSIQ for children with DCD, as suggested by Oliveras-Rentas et al. (2012) for an ASD population. While the European guidelines for diagnosing DCD suggest that IQ testing is not always necessary if academic achievement appears typical (Blank, Smits-Engelsman, Polatajko \& Wilson, 2011), the focus of this paper is on how to interpret such assessments when they are needed as well as how they impact on the diagnostic process. These results highlight the varied range of IQ profiles in those with DCD, and the need to consider the implications of an individual's particular performance profile, alongside their performance on standardized motor assessment and other aspects that are required in order for a diagnosis to be given. 
Although this study systematically examined the cognitive profile of children with DCD, limitations remain. Cognitive profiles were measured at one point in time. Performance may vary developmentally due to maturation and in response to intervention. Future work could examine peaks and troughs in cognitive abilities longitudinally and identify their association with outcome-related measures; whether it is motor performance in DCD or secondary consequences of DCD (e.g. academic achievement or psychosocial impact). This could also inform a typically-developing timetable of the cognitive and motor developmental trajectories. Second, combordity was not specifically tested. However, clinicians typically eliminate or identify other concerns if present during the diagnostic process and this type of information about other co-occurring diagnoses was collated from parents during the screening process. Further research may benefit from considering the impact of co-occurring diagnoses with DCD, as it is presumed that comorbidity will invariably alter the cognitive profile and, as a result, could highlight the specificity of the findings documented here.

\section{Conclusions}

A thorough assessment revealed that while children with DCD presented with weaker working memory and poorer processing speed, as measured by the Wechsler scales, than their typically developing peers, there is no primary deficit in these areas since a heterogeneous profile was seen across all intelligence domains. Individual difference analyses proved fruitful in addressing the research questions. The findings raise issues about practitioners identifying DCD based on a cognitive profile and suggest that each individual's cognitive strengths and weaknesses should be considered alongside their motor difficulties to determine appropriate intervention. Moreover, the motor confound in the PSI of the WISC means that these data should be treated with caution in a DCD population.

Acknowledgements. This research was jointly facilitated by an ESRC PhD studentship awarded to the second author and a Leverhulme Trust grant (RPG-2012-742) awarded to the final author. Special thanks go to all the children, parents, and schools that participated in the work described here. Thanks are also due to Dr Laura Crane for providing comments on the first draft of the manuscript.

\section{References}

Alloway, T. P. (2007). Working memory, reading and mathematical skills in children with developmental coordination disorder. Journal of Experimental Child Psychology, 96, 20-36.

Anderson, V. A., Anderson, P., Northam, E., Jacobs, R. \& Catroppa, C. (2001). Developmental of executive functions through late childhood and adolescence in an Australian sample. Developmental Neuropsychology, 20, 385-406.

American Psychiatric Association. (2013). Diagnostic and statistical manual of mental disorders (DSM, $5^{\text {th }}$ ed.). Arlington, VA: American Psychiatric Association.

Blank, R., Smits-Engelsman, B., Polatajko, H., \& Wilson, P. (2011). European Academy for Childhood Disability (EACD): Recommendations on the definition, diagnosis and 
intervention of developmental coordination disorder (long version). Developmental Medicine \& Child Neurology, 54, 54-93.

Castles, A., Kohnen, S., Nickels, L. \& Brock, J. (2014). Developmental disorders: what can be learned from cognitive neuropsychology. Philosophical Transactions of the Royal Society Biological Sciences, 369, doi:10.1098/rstb.2013.0407

Charman, T., Pickles, A., Simonoff, E., Chandler, S., Loucas, T. \& Baird, G. (2010). IQ in children with autism spectrum disorders: data from the Special Needs and Autism Project (SNAP). Psychological Medicine, 41, 619-627.

Dewey, D., Kaplan, B. J., Crawford, S. G. \& Wilson, B. N. (2002). Developmental coordination disorder: Associated problems in attention, learning, and psychosocial adjustment. Human Movement Science, 21, 905-918.

Geuze, R. H., Schoemaker, M. M. \& Smits-Engelsman, B. C. M. (2015). Clinical and research criteria for Developmental Coordination Disorder - should they be one and the same? Current Developmental Disorders Reports, 2, 127-130.

Goldstein, G., Allen, D. N., Minshew, N. J., Williams, D. L., Volkmar, F., Klin, A. \& Schultz, R. J. (2008). The structure of intelligence in children and adults with high functioning autism. Neuropsychology, 22, 301-312.

Gomez, A., Piazza, M., Jobert, A., Dehaene-Lambertz, G., Dehaene, S., \& Huron, C. (2015). Mathematical difficulties in developmental coordination disorder: Symbolic and nonsymbolic number processing. Research in Developmental Disabilities, 42, 167-178.

Grant, D. (2010). That's the way I think: Dyslexia, Dyspraxia and ADHD explained. Oxford, United Kingdom: Routledge.

Hagberg, B. S., Miniscalco, C. \& Gillberg, C. (2010). Clinic attenders with autism or attention-deficit/hyperactivity disorder: cognitive profile at school age and its relationship to preschools indicators of language delay. Research in Developmental Disabilities, 31, 1-8.

Henderson, S. E., Sugden, D. A., \& Barnett, A. L. (2007). Movement Assessment Battery for Children, 2nd Edition (Movement ABC-2). London: The Psychological Corporation

Kaplan, B. J., Wilson, B. N., Dewey, D. \& Crawford, S. (1998). DCD may not be a discrete disorder. Human Movement Science, 17, 471-490.

Kastner, J. \& Petermann, F. (2010). Developmental coordination disorder: relations between deficits in movement and cognition. Klinische Padiatrie, 222, 26-34.

Kirby, A. \& Sugden, D. A. (2007). Children with developmental coordination disorders. Journal of the Royal Society of Medicine, 100, 182-186.

Leonard, H. C. \& Hill, E. L. (2015). Executive difficulties in developmental coordination disorder: Methodological issues and future directions. Current Developmental Disorders Reports, 2, 141-149. 
Lingam, R., Hunt, L., Golding, J., Jongmans, M., \& Emond, A. (2009). Prevalence of developmental coordination disorder using the DSM-IV at 7 years of age: A UK population-based study. Pediatrics, 123, 693-700

Loh, P. R., Piek, J. P. \& Barrett, N. C. (2011). Comorbid ADHD and DCD: Examining cognitive functions using the WISC-IV. Research in Developmental Disabilities, 32, 1260-1269.

Mayes, S. D. \& Calhoun, S. L. (2008). WISC-IV and WIAT-II profiles in children with highfunctioning autism. Journal of Autism and Developmental Disorders, 38, 428-439.

Moura, O., Simoes, M. R. \& Pereira, M. (2014). WISC-III cognitive profiles in children with developmental dyslexia: specific cognitive disabilitiy and diagnostic utility. Dyslexia, 20, 19-37.

Oliveras-Rentas, R. E., Kenworthy, L., Roberson, R. B., Martin, A. \& Wallace, G. L. (2012). WISV-IV profile in high-functioning autism spectrum disorder: Impaired processing speed is associated with increased autism communication symptoms and decreased adaptive communication abilities. Journal of Autism and Developmental Disorders, 42, 655-664.

Parke, E. M., Thaler, N. S., Etcoff, L. M. \& Allen, D. N. (2015). Intellectual profiles in children with ADHD and comorbid learning and motor disorders. Journal of Attention Disorders, 1-10, Online First DOI: 10.1177/1087054715576343

Piaget, J. (1952). The origins of intelligence in children. New York: Norton \& Company.

Piek, J. P., Dyck, M. J., Francis, M. \& Conwell, A. (2007). Working memory, processing speed, and set-shifting in children with developmental coordination disorder and attention-deficit-hyperactivity disorder. Developmental Medicine and Child Neurology, 49, 678-683.

Pfeiffer, S. I., Reddy, L. A., Kletzel, J. E., Schmelzer, E. R. \& Boyer, L. M. (2000). The practitioner's view of IQ testing and profile analysis. School Psychology Quarterly, 15, 376-385.

Querne, L., Berguin, P., Vernier-Hauvette, M. P., Fall, S., Deltour, L., Meyer, M. E. \& de Marco, G. (2008). Dysfunction of the attentional brain network in children with Developmental Coordination Disorder: a fMRI study. Brain Research, 9, 89-102.

Smits-Engelsman, B. C. M \& Hill, E. L. (2012). The relationship between motor coordination and intelligence across the IQ range. Pediatrics, 130, 2011-3712.

Smits-Engelsman, B., Schoemaker, M., Delabastita, T., Hoskens, J. \& Geuze, R. (2015). Diagnostic criteria for DCD: Past and future. Human Movement Science, 42, 239-306.

Van der Fels, I. M., Te Wierike, S. C., Hartman, E., Elferink-Gemser, M. T., Smith, J. \& Visscher, C. (2015). The relationship between motor skills and cognitive skills in 4-16 year old typically developing children: A systematic review. Journal of Science and 
Medicine in Sport, 18, 697-703.

Wechsler, D. (2003). Wechsler intelligence scale for children - Fourth edition (WISC-IV). San Antonio, TX: The Psychological Corporation.

Wilson, P. H. \& McKenzie, B. E. (1998). Information processing deficits associated with developmental coordination disorder: A meta-analysis of research findings. Journal of Child Psychology and Psychiatry, 39, 829-840.

Figure 1. Variation of scores on the WISC indices $\quad$-DCD $-T D$

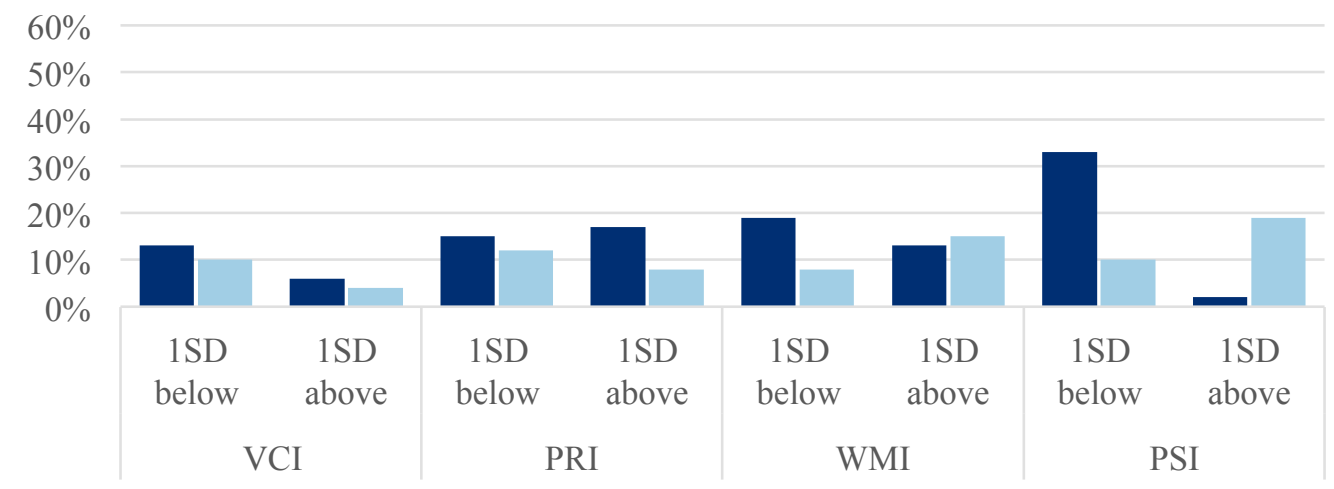


Figure 2. Variation of scores on the WISC subtests
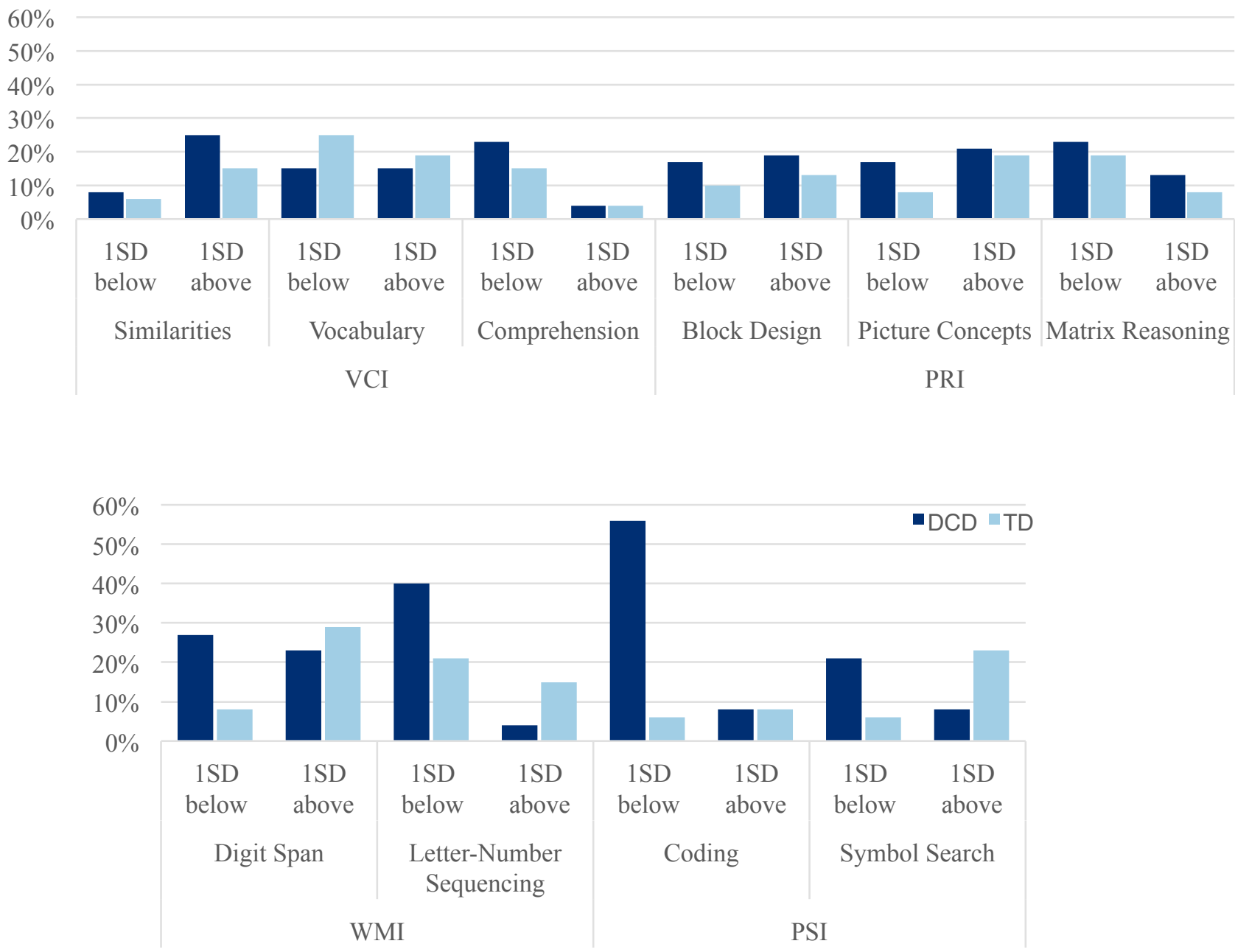